\title{
EFECTOS DE EXTRACTOS ACETÓNICOS Y ACUOSOS DE RICINUS COMMUNIS (RICINO), MELIA AZEDARACH (PARAÍSO) Y TRICHILLIA GLAUCA (TRICHILLIA), SOBRE LA HORMIGA NEGRA COMÚN (ACROMYRMEX LUNDI) ${ }^{1}$
}

\author{
EFFECTS OF ACETONIC AND WATER EXTRACTS OF RICINUS COMMUNIS, \\ MELIA AZEDARACH $Y$ TRICHILLIA GLAUCA ON BLACK COMMON \\ CUTTING ANT (ACROMYRMEX LUNDI)
}

\author{
Patricia Caffarini ${ }^{2}$; Paola Carrizo ${ }^{2}$; Alicia Pelicano ${ }^{2}$; Patricia Roggero; José Pacheco
}

\begin{abstract}
RESUMEN
Las hormigas cortadoras son importantes plagas en zonas agroforestales. A fin de sustituir los plaguicidas sintéticos empleados para su control por otros naturales, más degradables y selectivos y menos agresivos para el ambiente, se estudian diferentes especies de plantas que ocasionan variaciones comportamentales o tóxicas para esta plaga o su hongo simbionte. Sobre la hormiga negra común, se ensayó en laboratorio la respuesta a extractos acetónicos -obtenidos con Soxhlet-y acuosos -obtenidos por cocimientode ricino, paraíso y trichillia. En hormiguero artificial, en un ensayo sin libre elección, se ofrecieron discos de hojas de eucalipto topicados con dichos extractos $(n=3)$ y se registró el tiempo de acarreo de todos los discos, por tratamiento. Los extractos de ricino y paraíso generaron mayor efecto repelente, independientemente del solvente de extracción utilizado. Las pruebas de Friedman resultaron: extractos acuosos: $\mathrm{T}^{2}=85 ; p=0,0001$; acetónicos: $\mathrm{T}^{2}=5,50 ; p=0,0371$; por lo tanto, se obtuvo una diferenciación más clara entre los tratamientos para los extractos acuosos. Se probó entonces para los extractos acetónicos, el efecto tóxico por ingestión en confinamiento. En cajas de Petri se suministró a las hormigas estos extractos con dieta base artificial (n=5) y se registró la supervivencia diaria, ajustando las curvas a Weibull, obteniendo $\alpha$ (tasa media de mortalidad) y $\beta$ (supervivencia media) y comparando la longevidad máxima por ANOVA y Tukey. Ambos análisis resultaron coincidentes y el extracto de trichillia -menos repelentepresentó la mayor toxicidad por ingestión. El uso integrado de los extractos en campo permitiría aprovechar el efecto repelente del extracto de ricino para la protección del cultivo y el efecto tóxico del extracto de trichillia para el control de hormigueros.
\end{abstract}

Palabras clave: Ricino, paraíso, trichillia, hormiga cortadora.

\section{ABSTRACT}

Leaves cutting-ants are important pest in agroforestry. Many plant species are studied in order to replace synthetic pesticides, since they can produce behavior changes or toxic effects on the ants and on its symbiotic fungus. In lab trials, with the black common cutting ant, its response to acetone and water extracts of M. azedarach, T. glauca, and R. communis was assayed. In artificial nest $(n=3)$ without chance of choice, they were offered pieces of eucalyptus leaves with extracts applied on. Carrying time by treatment was registered. Pieces with ricinus and melia (acetone and water extracts) were the least accepted-highest repellency effect-according to Friedman tests (water $T 2=85, p=0,0001$; acetone $T 2=5,50, p=0,0371$ ). However, water extracts performed a most definite separation within. Then, toxic effects for ingestion from acetone extracts were tested in vitro. In Petri dishes, acetone extracts in basic diet were offered to the ants $(n=5)$ and daily survivors were registered. Weibull function was adjusted to obtain $\mathbb{\nabla}$ (mean rate of mortality) and $\beta$ (daily survivorship); besides, maximum longevity was compared by means of ANVA and Tukey. Both analysis obtained the same results, and trichillia extract -the least repellent-caused the highest toxic effect. That suggests taking advantage of both outdoors, and to integrate the repellent effect of ricinus in the crops and the toxic effect of trichillia in nests.

Key words: ricinus-melia-trichillia-black cutting ant

1 Proyecto UBACyT G 062. Bioinsumos para el manejo de plagas.

2 Cátedra de Zoología Agrícola. Facultad de Agronomía. Universidad de Buenos Aires. Av. San Martín 4453 C1417DSE. Buenos Aires, Argentina. E-mail: caffarin@agro.uba.ar

Fecha de Recepción: 12 Diciembre 2006

Fecha de Aceptación: 08 Junio 2007 


\section{INTRODUCCIÓN}

Las hormigas -Familia Formicidae (Orden: Hymenoptera)- son insectos polífagos, siendo algunas especies importantes plagas de la agricultura por los daños económicos que causan (Forti y Castellani Boaretto, 1997). La subfamilia Myrmicinae es la más abundante en especies y presenta la mayor diversidad de hábitos (Fowler et al., 1991); en ella, las hormigas cortadoras (tribu Attini) colectan material vegetal que utilizan como sustrato para el desarrollo del hongo simbionte, que es alimento para larvas y adultos (Da Silva et al., 2001). Estas hormigas se encuentran ampliamente distribuidas en la Argentina: Acromyrmex lundi u hormiga negra común es una de las especies más difundidas en las zonas agroforestales del país, extendiéndose desde el norte argentino hasta el paralelo $44^{\circ} \mathrm{S}$ (Kusnezov, 1978; Farji Brener y Ruggiero, 1994). Habitualmente, se ha recurrido para su control a productos tóxicos de síntesis, involucrados en su mayoría con efectos adversos causados al ambiente u otros organismos no blanco. Desde la prohibición del uso de los insecticidas clorados hay un creciente interés en la investigación sobre productos eficaces, más selectivos y ambientalmente menos agresivos (Baird, 2001). Así, han sido estudiadas diversas especies de plantas que presentan actividad repelente, disrupción comportamental o tóxicas para las hormigas y/o inhibitorias del hongo simbionte (Hebling et al., 2001), debido a la presencia de metabolitos secundarios como alcaloides, terpenos, fenoles y aceites esenciales.

En algunas especies de Meliáceas como Azadiractha indica y Melia azedarach se ha detectado la presencia de compuestos de acción insecticida como azadiractina y salanina. Varias especies del género Trichillia (Meliaceae) contienen tetranortriterpenoides en sus raíces, con actividades disuasivas sobre varios insectos (Simmonds et al., 2001). Pelicano et al. (2002) demostraron efectos de toxicidad en coleópteros, causados por extractos de Trichillia glauca, conteniendo limonoides como trichillina. Silveira et al. (2003) observaron un alto potencial insecticida por ingestión, para hormigas cortadoras en dos especies de Trichillia, sobre operarias de Atta sexdens rubropilosa. El alcaloide ricina, extraído de la hoja de Ricinus communis (Euphorbiaceae), mostró alta toxicidad para operarias de Atta sexdens (Acácio-Bigi, 1997).

\section{OBJETIVO}

Estudiar los efectos que presentan extracciones acetónicas y acuosas de Ricinus communis (ricino), Melia azedarach (paraíso) y Trichillia glauca (trichillia) sobre la hormiga negra común (Acromyrmex lundi).

\section{MATERIALES Y MÉTODOS}

Todos los experimentos se realizaron en laboratorio, en condiciones controladas a $24 \pm 2{ }^{\circ} \mathrm{C}$; $70-80 \%$ HR y $12 \mathrm{hrs}$. de fotoperíodo.

\section{OBTENCIÓN DE EXTRACTOS VEGETALES (RICINO, PARAÍSO Y TRICHILLIA):}

Los extractos acetónicos: se obtuvieron mediante Soxhlet a partir de hojas de cada especie previamente picadas y secadas en estufa.

Los extractos acuosos: se obtuvieron por cocimiento en agua de hojas de cada especie picadas.

\section{Experimento A}

En hormiguero artificial (Della Lucia, 1993) se plantearon dos ensayos sin libre elección con todos los extractos. Para ello se topicaron hojas del tercio superior de plantines de 6 meses de edad de Eucalyptus camaldulensis -especie preferida por las hormigas cortadoras (Vendramin et al., 1995)-. De ellas se extrajeron con sacabocado 15 discos de $5 \mathrm{~mm}$ de diámetro colocados en tres grupos, que se ofrecieron en la playa de forrajeo del hormiguero. Se realizaron tres repeticiones $(n=3)$ a intervalos de 48-72 hrs. Entre los ensayos se proporcionó a las hormigas la dieta habitual: hojas de ligustro y rosal, avena y piel de frutos cítricos.

Observando periódicamente la playa de forrajeo, se registró el tiempo de acarreo final (tiempo que las obreras emplearon en recoger todos los discos). Los resultados se analizaron mediante Friedman y pruebas a posteriori $($ alfa $=0,05)($ Conover, 1980) utlizando el software Infostat (1994).

\section{Experimento B}

Se llevó a cabo en caja de Petri ( $n=5$, cada una con 6 hormigas) con provisión de agua y dieta base sólida (Pelicano et al., 1999) a la que se adicionaron los extractos acetónicos de ricino, trichillia y melia o ningún extracto (testigo). Se llevó a cabo el recuento 
diario de hormigas sobrevivientes en la caja y las curvas obtenidas se ajustaron a la distribución de Weibull (Daniel, 1978). Del ajuste se obtuvieron las variables de la función: $\alpha$, que representaría la tasa media de mortalidad de la población y $\beta$, que representaría su supervivencia media. Se comparó la longevidad máxima de los tratamientos, mediante ANOVA y prueba a posteriori (Tukey) $($ alfa $=0,05)$ (Zar, 1999).

\section{RESULTADOS Y DISCUSIÓN}

\section{EXPERIMENTO A}

En la Figura 1 fueron graficados los resultados correspondientes al experimento.

Los trozos de hoja de eucalipto topicados con extractos acetónicos y acuosos de ricino y paraíso fueron los menos aceptados -efecto de repelenciamientras que, tanto la trichillia como el testigo, presentaron tiempos de acarreo menores (prueba de Friedman para extractos acuosos: $\mathrm{T}^{2}=85 ; p=0,0001$; para extractos cetónicos: $\left.\mathrm{T}^{2}=5,50 ; p=0,0371\right)$.

Este efecto de repelencia que produjo el extracto de ricino a bajas concentraciones podría corresponderse con el efecto tóxico que hallaron Mendes Acacio et al. (1998) al ensayar la ingestión y topicación de dicho extracto sobre obreras de Atta sexdens rubropilosa. Efectos similares observaron Hebling et al. (1996) y Kitamura et al. (1999). Contrariamente a lo observado para varios insectos por Simmonds et al. (2001), los extractos de trichillia no parecieron influir en la respuesta de aceptación del material vegetal tratado en las concentraciones ensayadas.

La tendencia en la respuesta a los extractos coincidió para ambos solventes de extracción, resultando los de ricino y paraíso los más efectivos como repelentes. Sin embargo, en la respuesta de repelencia se obtuvo una diferenciación más clara para los extractos acuosos respecto de los extractos acetónicos. Estos últimos fueron entonces probados in vitro, para determinar su efecto tóxico por ingestión.

\section{EXPERIMENTO B}

En la Figura 2 se detalló el progreso en las curvas de supervivencia para las hormigas expuestas a la dieta con extractos.

La pendiente de la curva de supervivencia diaria debida al tratamiento con trichillia es más pronunciada, mientras que las curvas correspondientes a los demás extractos no se diferenciaron tan netamente de aquella del testigo, aunque son relativamente peores, ya que implican una mayor tasa de mortalidad y una menor longevidad total (ver

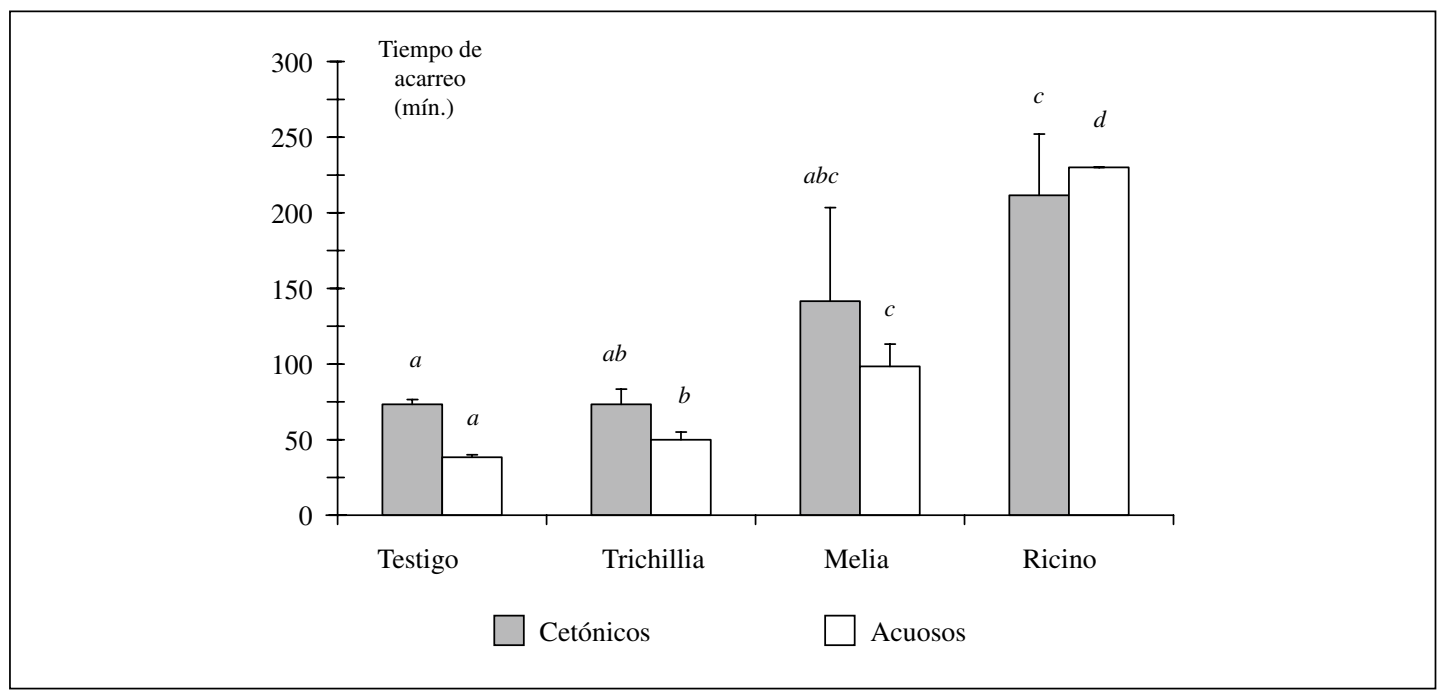

Figura 1. Resultados para tiempo de acarreo para los extractos cetónicos y acuosos de Trichillia, Melia, Ricinus y Testigo (solvente de extracción). Se representaron los promedios y el error estándar de la media. Las letras representan diferencias en la suma de rangos para las pruebas a posteriori luego que la prueba de Friedman resultara significativa $($ alfa $=0,05)$ dentro de cada método (solvente de extracción). 


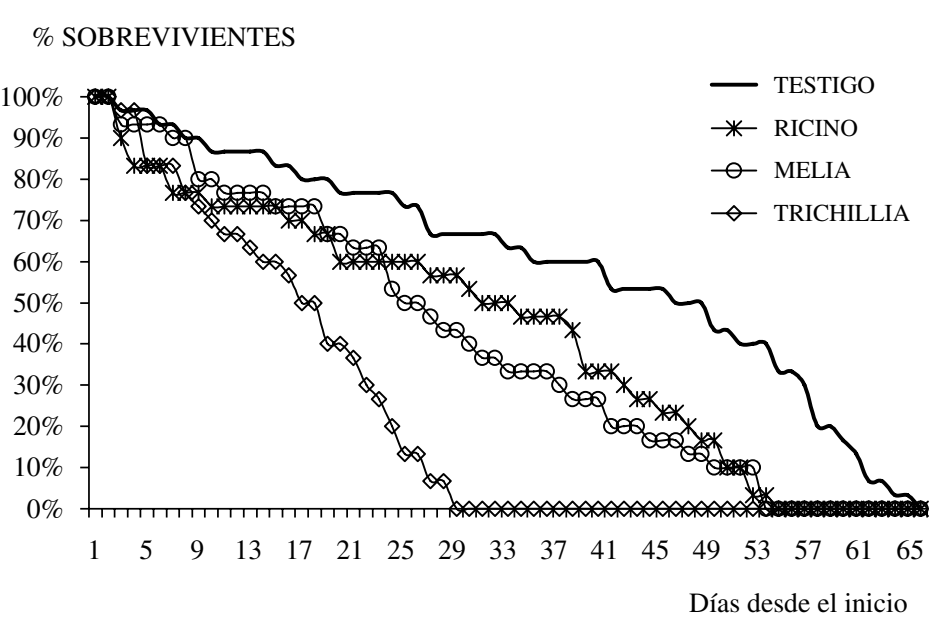

Figura 2. Progreso diario en la sobrevivencia de hormigas alimentadas con dieta y extractos acetónicos.

Tabla 1). Silveira et al. (2003) observaron efectos similares trabajando con dos especies de trichillia en bioensayos de ingestión en laboratorio con Atta sexdens rubropilosa.

La trayectoria de las curvas fue muy similar en la supervivencia diaria, entre ricino y paraíso, aunque resultaron en una reducción en la supervivencia máxima del 27 y $35 \%$, respecto del testigo. La prueba de ANVA, sin embargo, consideró significativa tal reducción (Figura 3) sólo en el caso del extracto cetónico de trichillia, cuando ésta disminuyó un $61 \%$ respecto del testigo (Tabla 1 ).

En la Tabla 1 a continuación se muestran las variables obtenidas en el ajuste.

Tabla 1

Variables obtenidas en el ajuste a la función de Weibull (curvas de supervivencia)

\begin{tabular}{|l|c|c|}
\hline \multicolumn{1}{|c|}{ Tratamiento } & $\alpha$ & $\beta$ \\
\hline Testigo & 1,886 & 49,334 \\
\hline Melia & 1,645 & 31,936 \\
\hline Ricino & 1,324 & 36,154 \\
\hline Trichillia & 1,900 & 19,296 \\
\hline
\end{tabular}

En el ajuste obtenido para la función que describe la curva de mortalidad, puede apreciarse que el efecto producido por el extracto de trichillia se origina en una alteración marcada en ambas variables. En la Figura 2 se aprecia que la longevidad máxima es inferior a la mitad de aquella del testigo, lo cual se corroboró con la prueba de ANVA para la variable, en la Figura 3. En la misma, se detallan los resultados de la prueba de Tukey para la supervivencia máxima, luego de obtener diferencias significativas para el ANVA ( $\mathrm{F}=28,11 ; p=0,0001)$.

Las respuestas de comportamiento e ingestión para los mismos extractos acetónicos resultaron invertidos. Así, el extracto de trichillia, que fue relativamente más tóxico en la dieta, fue también el menos repelente. Esto sugiere la posibilidad de integrar la utilización de los extractos, para que cumplan diferentes funciones según sus propiedades y la respuesta producida en la población de hormigas. Su uso integrado podría mejorar los resultados a campo para el manejo de esta plaga. $\mathrm{La}$ siguiente etapa de investigación deberá focalizarse entonces en las pruebas de campo, donde podrá considerarse la repelencia para el acarreo que produce el extracto de ricino en el cultivo y el efecto tóxico del extracto de trichillia para la fabricación de cebos con productos naturales, a utilizar en los hormigueros (Caffarini et al., 2006). 


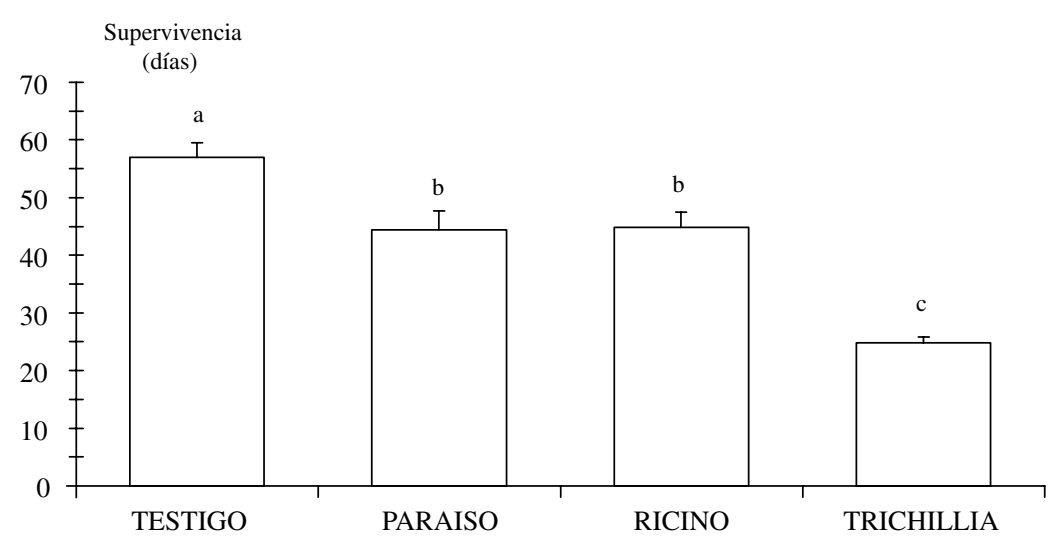

Figura 3. Supervivencia máxima (días) para hormigas alimentadas con dieta y extractos cetónicos. Se representan los promedios y el error estándar de la media. Las letras representan el ordenamiento entre las medias a partir de la prueba de Tukey una vez que la prueba de ANVA resultara significativa $($ alfa $=0,05)$.

\section{LITERATURA CITADA}

ACACIO-BIGI, M. F. M. 1997. Determinaçäo do efeito tóxico de extractos foliares e derivados químicos de Ricinus communis L. para operarias de Atta sexdens L. (Hymenoptera: Formicidae). Tese de Doutorado. Instituto de Biociencias. UNESP. Río Claro. 134 p.

BAIRD, C. 2001. Química ambiental. Parte II. Sustancias tóxicas. Cap. 6: Compuestos químicos tóxicos. Pp. 300-388. Ed. Reverte SA. Barcelona. España. 622 pp.

CAFFARINI, P.; P. CARRIZO; A. PELICANO. 2006. Extractos cítricos como atrayentes para hormiguicidas con sustancias naturales. Revista de la Facultad de Ciencias Agrarias de la Universidad Nacional de Cuyo. Mendoza, 1: 19-26.

CONOVER, W.J. 1980. Practical non-parametric statistics. Wiley, NY.

DA SILVA, M. F. DAS G. F.; VICTOR, S. R.; RODRÍGUEZ -GAMBOA, T.; BELLINI, A.; MAFEZOLI, J.; GUERREIRO, G.; AMBROZI, A. R. P.; BIOVATTI, M. W.; FERNÁNDEZ, J. B.; VIEIRA, P. C.; CASTRO, O. C.; PAGNOCCA, F. C.; BUENO, O. C.; HEBLING, $M$. J. A. 2001. Integraçao entre plantas e formigas cortadeiras: Perspectivas de utilização de plantas para o controle: Sistemática micromolecular da Ordem Rutales e suas implicaçoes frente ao fungo Leucoagaricus gongylophorus. Anais do XV Encontro de Mirmecologia 2001 - Londrina -Brasil, pp. 105-108.

DANIEL, D.W. 1978. Applied nonparametric statistics. Houghton Mifflin Co., USA. 503 pp.

DELLA LUCÍA, T.M.C. (ED) 1993. As formigas cortadeiras. Viçosa, Mina Gerais. Brasil. 262 pp.

FARJI BRENER, A.G.; A. RUGGIERO. 1994. Leaf-cutting ants (Atta and Acromyrmex) inhabiting Argentina: patterns in species richness and geographical range sizes. J. Biogeography 21: 390 - 391 .

FORTI, L.C.; CASTELLANI BOARETTO. 1997. Formigas cortadeiras. Biología, ecología, danos e controle. Botucatu. SP.61 pp.
FOWLER H.G.; L.C. FORTI; C.R.F. BRANDAO; J.H.C. DELABIE E H.L.VASCONCELOS. 1991. En Ecología nutricional de insectos e suas implicações no manejo de pragas. A. Panizzi \& J. Parra. Ed. Manole Ltda. 359 pp. São Paulo.

hebling, M. J. A.; PAGNOCCA, F. C.; BUENO, O. C.; FERNANDES, J. B.; BACCI JR, M.; VIEIRA, P. C.; DA SILVA, M. F. 2001. Compuestos extraidos de plantas con perspectivas de utilizacion en el control de hormigas cortadoras, Anais do XV Encontro de Mirmecologia 2001 - Londrina - Brasil.

HEBLING, M. J.; BUENO, O. C.; PAGNOCCA, F. C.; BACCI JR., M.; FERNÁNDEZ, J.B.; VIEIRA P.C. 1996. A utilizaçao de produtos naturais para o controle de formigas cortadeiras e ou fungo simbionte. Rev. Naturalia, 24 (n. esp.): 311-313.

INFOSTAT, VERSIÓN 2004. Manual del Usuario. Grupo InfoStat, FCA, Universidad Nacional de Córdoba. 1ra Ed., Editorial Brujas. Argentina.

KITAMURA A.E.; HEBLING, M.J.; TAKAMASHI DEL BIANCO, M.; BUENO, O.C.; PAGNOCCA, F.C.; BACCI JR., M.; FERNÁNDEZ, J.B.; VIEIRA, P.C. 1999. Determinação da toxicidade da ricinina para operarias de Atta sexdens L.. (Hymenoptera, Formicidae) em laboratorio. Rev. Naturalia 24 (n. esp.): 307-309.

KUSNEZOV, N. 1978. Hormigas argentinas: claves para su identificación. Miscelánea (Tucumán, Argentina) 61 pp.

MENDES ACACIO-BIGI, M. F.; HEBLING. M. J.; BUENO, C. O.; PAGNOCCA, F. C.; DA SILVA, O. A.; FERNÁNDEZ, J. B.; VIEIRA, P. C. 1998. Toxicidade de extractos foliares de Ricinus communis L. para operarias de Atta sexdens rubropilosa Forel, (Hymenoptera, Formicidae). Revta. Bras. Ent. 41 (2-4) : 239-243.

Pelicano, A.; CAFFARINI, P.; CARRIZO, P. 1999. Sobrevivencia de operarias de Acromyrmex lundi y A. striatus 
aisladas de hormigueros y alimentadas con dieta artificial. IDESIA 17-91:99. Universidad de Tarapacá. Chile.

PELICANO, A.; CAFFARINI, P.; CARRIZO, P.; FERRO, M. 2002. Eficacia de sustancias de origen vegetal sobre hormigas cortadoras. XV Jornadas Fitosanitarias Argentinas. Río Cuarto. Córdoba.

SILVEIRA, C.; HEBLING, M.J.A.; ROCHA, W.C.; FERNANDES, J.B.; PAGNOCCA, F. C.; BUENO, O. C.; BACCI JR., M.; VIEIRA, P.; SILVA, M. F. 2003. Toxicidade de duas especies do género trichillia para operarias de Atta sexdens rubropilosa (Hymenoptera: Formicidae). Anais do XVI Simposio de Mirmecología. Florianopolis. Sc. P.
SIMMONDS, M.S.; STEVENSON, P.C.; PORTER, E.A.; VEITCH, N.C. 2001. Insect antifeedant activity of three tetranortriterpenoides form Trichillia pallida J. Natural products, Columbus. 64, 8: 1117-1120.

VENDRAMIN, J. D.; SILVEIRA, NETO, S.; CERIGNONI, J.A. 1995. Nao Preferencia de Atta sexdens rubropilosa Forel (Hymenóptera: Formicidae) por especies de Eucaliptus. Ecosistema, 20: 87-92.

ZAR, J.H. 1999. Biostatistical analysis. 4th ed. Prentice-Hall, Inc. Simon and Schuster/A Viacom Co. New Jersey. USA. $663 \mathrm{pp}$. 